Note: This is a preprint of a paper submitted for publication. Contents of this paper should not be quoted or referred to without permission of the author(s).

To be presented at Time-Resolved Electron and X-Ray Diffraction Conference,

San Diego, California, July 9-14, 1995 and published in proceedings of the conference by SPIE (Society of Photo-Optical Instrumentation Engineers, Bellingham, Washington)

\title{
TIME-RESOLVED MATERIALS SCIENCE OPPORTUNITIES USING SYNCHROTRON X-RAY SOURCES
}

\author{
B. C. Larson and J. Z. Tischler
}

Solid State Division, Oak Ridge National Laboratory

P.O. Box 2008, Oak Ridge, Tennessee 37831-6030

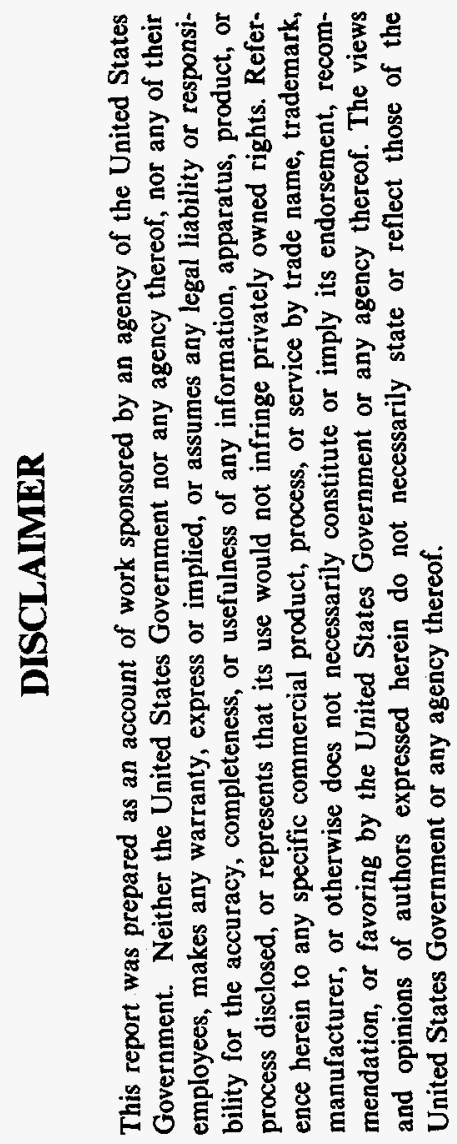

\begin{abstract}
"The submitted manuscript has been authored by a contractor of the U.S. Government under contract No. DE-AC05-840R21400 Accordingly, the U.S. Government retains : nonexclusive, royalty-free license to publish or reproduce the published form of this contribution, or allow others to do $s 0$, for U.S Government purposes.
\end{abstract}

\author{
Prepared by \\ SOLID STATE DIVISION \\ OAK RIDGE NATIONAL LABORATORY \\ Managed by \\ LOCKHEED MARTIN ENERGY SYSTEMS \\ under \\ Contract No. DE-AC05-84OR21400 \\ with the \\ U.S. DEPARTMENT OF ENERGY \\ Oak Ridge, Tennessee
}

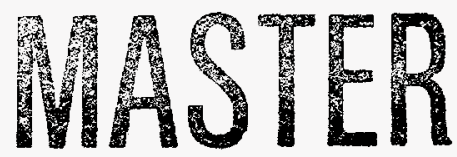

June 1995 


\section{DISCLAIMER}

Portions of this document may be illegible in electronic image products. Images are produced from the best available original document. 


\title{
Time-Resolved Materials Science Opportunities Using Synchrotron X-Ray Sources
}

\author{
B. C. Larson and J. Z. Tischler \\ Solid State Division, Oak Ridge National Laboratory \\ P.O. Box 2008, Oak Ridge, Tennessee 37831-6030
}

\begin{abstract}
The high brightness, high intensity, and pulsed time-structure of synchrotron sources provide new opportunities for time-resolved $x$-ray diffraction investigations. With third generation synchrotron sources coming on line, high brilliance and high brightness are now available in $x$-ray beams with the highest flux. In addition to the high average flux, the instantaneous flux available in synchrotron beams is greatly enhanced by the pulsed time structure, which consists of short bursts of $x$-rays that are separated by $~$ tens to hundreds of nanoseconds. Timeresolved one- and two-dimensional position sensitive detection techniques that take advantage of synchrotron radiation for materials science $x$-ray diffraction investigations are presented, and time resolved materials science applications are discussed in terms of recent diffraction and spectroscopy results and materials research opportunities.
\end{abstract}

Keywords: Synchrotron, $x$-ray, time-resolved, CCD detector, microchannel plate, laser, thin-film, melting, Mössbauer, phonon

\section{NTRODUCTION}

Synchrotron $x$-ray sources are characterized by beams with high flux, a continuous energy spectrum, narrow angular widths, pulsed time-structure, and a well defined polarization state. These $x$-ray beam aspects have been utilized in widely varying fields (biology, geology, physics, chemistry, materials science, and others) to develop new techniques and perform investigations that in most cases could not have been carried out using conventional $x$-ray generators. The high flux of synchrotron sources is useful for all types of $\mathbf{x}$-ray diffraction and spectroscopy investigations, making high precision measurements possible and making all measurements less time consuming. The high flux and high angular resolution have been particularly beneficial for macromolecular crystallography due to the short lifetime of irradiated crystals and signal-to-noise improvements. The continuous spectrum has made anomalous scattering crystal structure determinations for biological as well as physical science areas a much more general technique. In addition the continuous spectrum has led to general use of extended $x$-ray absorption fine structure (EXAFS) and the related techniques of $x$-ray absorption near edge structure (XANES), diffraction anomalous fine structure (DAFS), and diffraction anomalous near edge structure (DANES) for local structural and chemical investigations. The availability of circularly polarized radiation from bend magnet beams and the development of other methods for generating right and left handed circularly polarized beams has been particularly useful for magnetic $x$-ray scattering and absorption investigations. The overwhelming majority of investigations are made under equilibrium or kinetically frozen conditions, where the structure or state of the material under investigation is, to the extent possible, kept from changing during the measurement. For these static or steady state measurements, the pulsed time structure of synchrotron $x$-ray beam plays no role.

However, the pulsed time-structure combined with the high flux is important for investigations carried out under transient conditions to determine the rate at which processes evolve and new structures are formed, and for investigations involving time dependent aspects such as Mössbauer spectroscopy. Time resolved investigations are the primary focus of this paper. Such investigations not only require detailed measurements, they also require that the measurements be carried out on a time-scales short with respect to the time for significant structural changes to occur or short with respect to spectroscopic lifetimes. 


\section{TIME SCALES OF MEASUREMENTS}

The requirements of time-resolved measurements vary considerably; whereas study of the exceedingly complex reactions associated with the aging of cement to form concrete may require measurements with time resolution of thousands of seconds to days or weeks, investigations of phenomena such as electron-phonon interactions associated with laser melting of semiconductors or metals require time resolution in the picosecond range. These materials science examples represent time scales differing by 15 orders of magnitude, and they have analogs in other fields as well, such as picosecond time scale of some aspects of the photosynthesis process compared to the long time scales associated with diffusion in plants. Figure 1 lists matcrials science phenomena spanning these widely varying times scales, also indicated are detection schemes appropriate for addressing the various timescales and a catigorization of modes of measurement as governed by the pulse spacings and the pulse widths of synchrotron radiation.

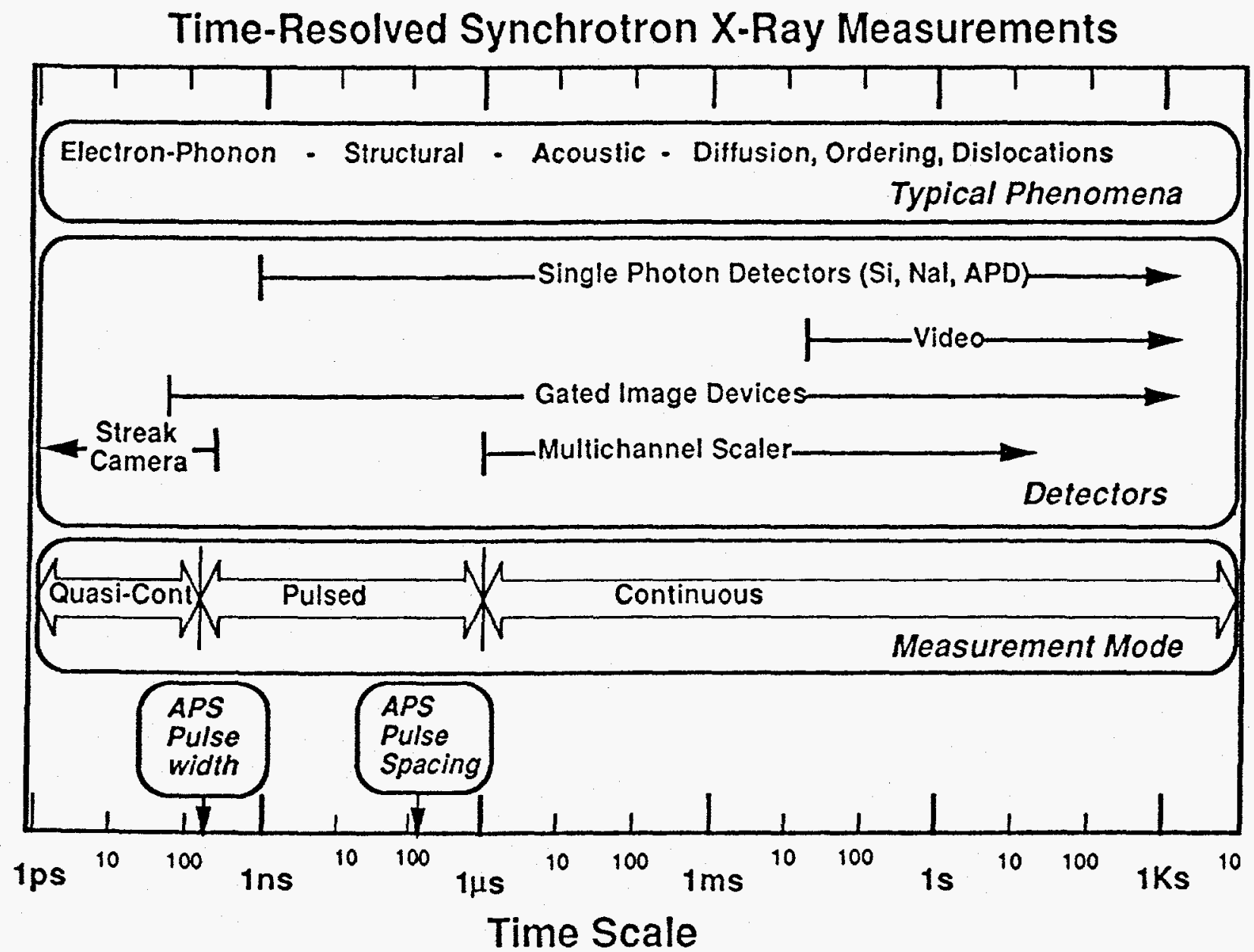

Fig. 1. Overview of time-scales and time-resolved detection techniques for materials science investigations

For time scales greater than a few microseconds, synchrotron beams may be considered continuous and measurements may be performed using arbitrary time slices without regard for the pulsed nature of the source. For these time scales, time resolved techniques using synchrotron sources similar to those using conventional $x$-ray sources are applicable, but they have the advantages of higher incident beam fluxes, wavelength tuneability, and angular collimation. On the other hand, the pulsed time structure of the incident $x$-rays is an important aspect below the microsecond regime. The temporal spacing of these sharp pulses allows measurements to be synchronized with individual $x$-ray pulses to achieve very high time resolution, analogous to time resolved measurements using electron discharge or pulsed-laser $x$-ray sources. On the one-to-ten picosecond scale, 
synchrotron $x$-ray pulses again become continuous, or quasi-continuous, with varying, but well known time structure. Although, to our knowledge, such measurements have not been attempled using hard $x$-rays, some work has been carried out in the VUV region using a streak camera. Because electron bunch jitter is likely to be the order of a few picoseconds, resolution below this level is apparently not possible with present synchrotrons.

\section{TUMIE RESQLYED TECHNIQUES}

For time resolved measurements with resolution $\sim 30$ milliseconds, where the synchrotron pulses appear as a continuous stream of $x$-rays, experiments need only be concerned with the time scale of the phenomena to be studied, and time resolved measurements in this time range do not differ from measurements using conventional $x$-ray sources. Figure $2 a$ illustrates the use of video based $2 \mathrm{D}$ detector systems to make direct measurements with -17 ms time frames. As depicted in Fig. $2 b$, single detectors can be used to take arbitrary size time slices simply by electronically sampling detector counts or through the use of multi-channel scaling techniques. It should be noted that avalanche photo-diodes (APD) as single detectors have the capability of sub-nanosecond resolution so they can be used for exceedingly high count rates. Taking advantage of the photon energy sensitivity and dual counting chains, it is in principle possible to count multiple photons per synchrotron pulse.

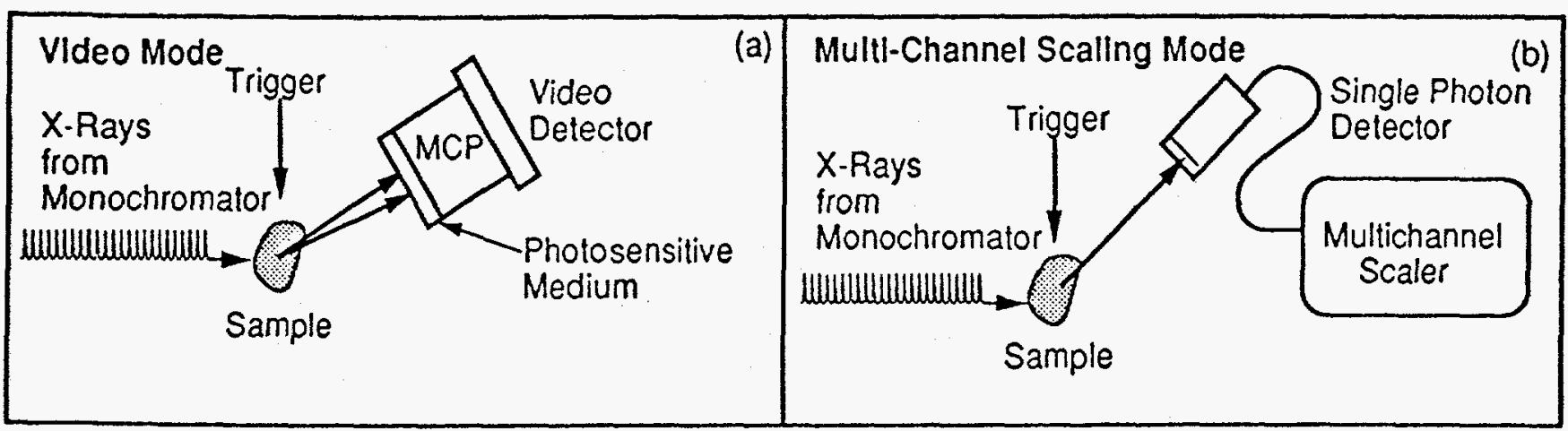

Fig. 2. Scattering configuration (a) for time resolved measurements in the long time-scale using video mode; and (b) for time-resolved scattering measurements made using a single detector and a multichannel scaler.

The pulsed nature of synchrotron $x$-rays becomes a factor in considering time-scales from microseconds to nanoseconds, and plays a major role in determining measurement techniques. Since $x$-ray pulses probing the sample arrive at discrete times, it is necessary to synchronize the phenomena to be studied with the arrival time of individual $x$-ray pulses. This limits the number of measurements that can be made during any one triggering cycle of the phenomena to -200 ns spacings, but the $x$-ray pulses deliver all of the $x$-rays within $\sim 150$ picoseconds so that with $\sim 200$ ns timing resolution one obtains measurements made within $\sim 150$ picoseconds. For strongly scattering phenomena where more than one $x$-ray is scattered from a single $x$-ray pulse, it is necessary to provide either an integrating position sensitive detector such as a CCD device or make use of a single detector that analyzes the pulse height to determine the number of scattered photons per $x$-ray pulse. It is necessary for the detector to measure consecutive synchrotron pulses without pile-up or to provide a gate for the photomultiplier. Examples of these techniques can be found in pulsed laser investigations of Larson et al ${ }^{1,2}$ in the high scattering rate regime and Bartunik ${ }^{3}$ in the low scattering regime.

The picosecond time scale represents a frontier for synchrotron $x$-ray diffraction measurements that has yet to be realized. Although picosecond measurements have been carried out in the soft $x$-ray regime using streak cameras, ${ }^{4}$ work using hard synchrotron $x$-rays has not been reported. Figure 3 illustrates schematically a method which would utilize the continuous $x$-:ay flux over the $\sim 150$ picosecond duration of individual pulses. This continuous (or quasi-continuous) mode no longer uses the $x$-ray pulses as strobes, but rather provides continuous illumination for the hundred-fifty picosecond duration. As depicted in Fig. 3, the time resolution is once again delermined by the delector, and $x$-ray streak cameras provide resolution in the $<10$ picosecond range. 


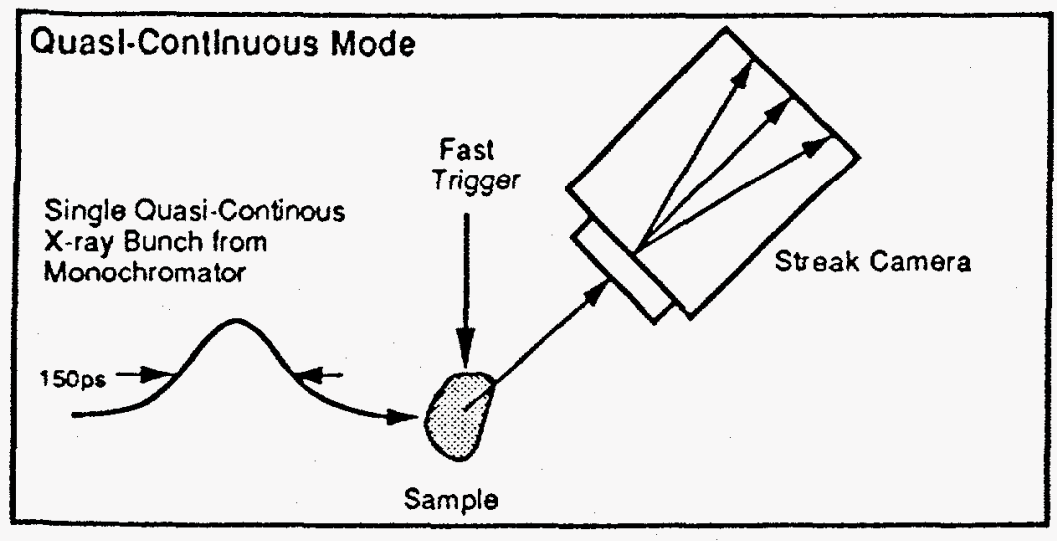

Fig. 3. Scattering configuration for quasi-continuous mode measurements in which a streak camera resolves scattering within a single synchrotron bunch. Account must be taken of the shape of the x-ray pulse in this mode.

\section{EXPERIMENTAL GEOMIETRY FOR 2D DETECTORS}

Significant gains have been made in the development of position sensitive $x$-ray detectors in terms of speed, resolution, and uniformity; however, detectors remain an area in which additional gains would be very helpful. We discuss three types of position sensitive detectors that have particular relevance to the materials science investigations in this paper. Figure 4 depicts the $x$-ray scattering geometry used by Clarke ${ }^{5}$ et al for time-resolved measurements of lattice strain in thin films and multilayers during thermal annealing. In this Lemonnier angular dispersive focusing geometry, ${ }^{6}$ a high resolution radial scan of the Bragg reflection of the substrate and film composite is dispersed along a narrow line on the detector. With the Texas Instruments virtual phase chip CCD detector consisting of 584 rows by 390 columns, $20 \mu \mathrm{s}$ per single row transfers of data out of the exposed region was possible for streak camera like continuous measurements of the Bragg scans. Although their measurements only required $\sim 100 \mathrm{~ms}$ transfers, the capability of rapid row transfers suggests an exciting possibility if the speed of the transfer can be increased to the point of $\sim 2 \mu \mathrm{s}$ per row. Since the single bunch mode of the Cornell High Energy Synchrotron (CHESS) corresponds to $2.46 \mu$ s between pulses, it would then be possible to collect scattering from a single $x$-ray bunch in each row. The time resolution would then be determined by the 150 ps width of the $x$-ray pulse rather than the row transfer rate.

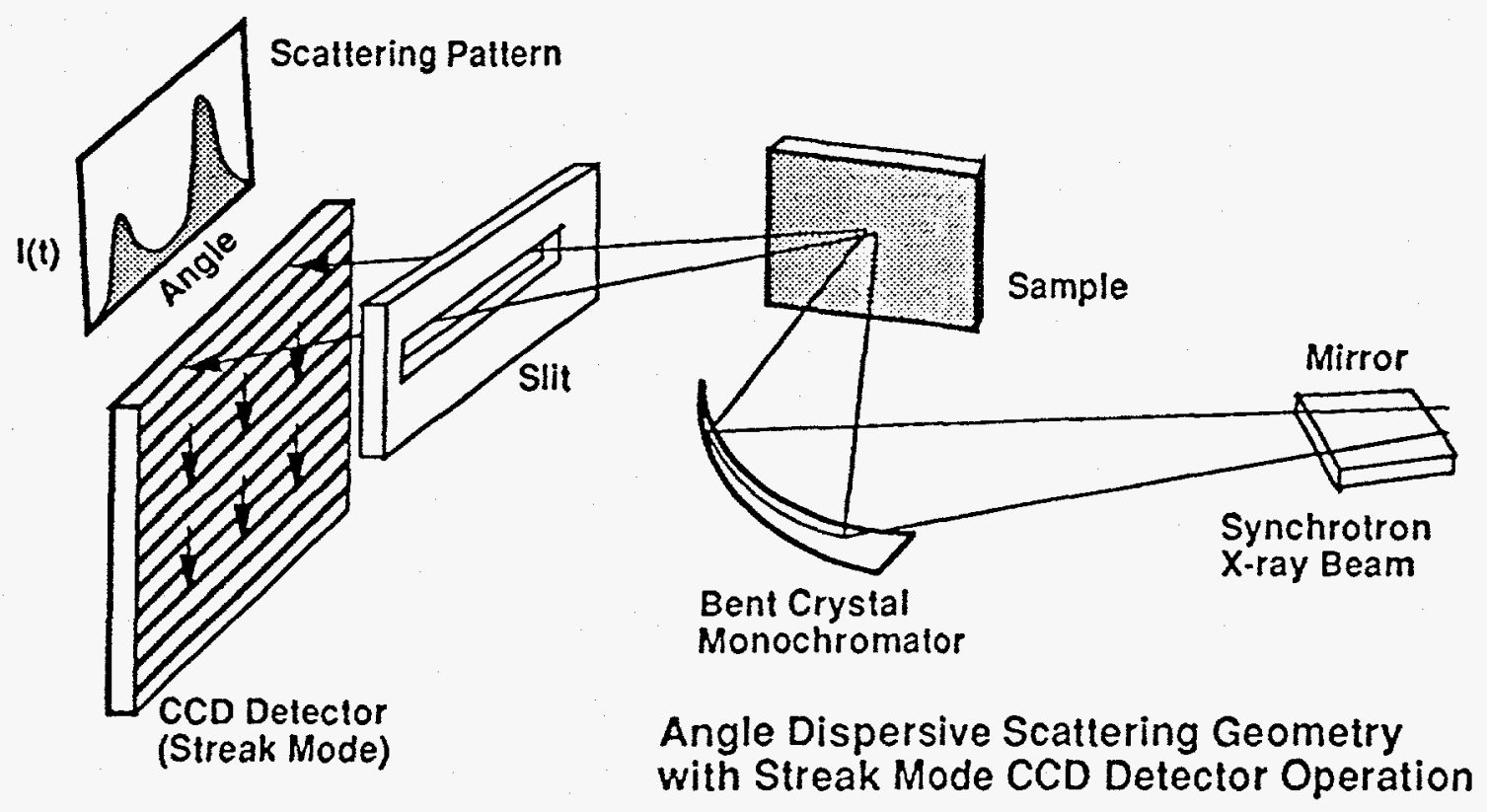

Fig. 4. Schematic drawing of the scattering geometry for an angular dispersive focusing monochromator and a CCD camera operated in a streak mode. The slit limits exposure of the CCD to a single row (or a few rows) and the data are continuously transferred down the detector. 
Figure 5a shows a gated microchannel plate $2 \mathrm{D}$ detector. ${ }^{7}$ Such a detector is capable of seiecting single synchrotron pulses from a stream of pulses, excluding scattering from previous and following prulses. Cold is used as a photocathode in combination with a pulsed microchannel plate and optical CCD recording. High voltage pulses as short as $5 \mathrm{~ns}$ can be selected so that phenomena triggered for individual synchrotron $\mathrm{x}$-ray pulses can be measured without having the noise and scattering from other $x$-ray pulses. As shown in the Fig. 5a, the shortest MCP pulses are achieved through the use of an auxiliary $-1 \mu \mathrm{sec}$ pedestal, on which the short pulse is added. Typical measurements with a detector of this nature would involve a fast transient stim:ilus such as a laser pulse striking a sample at a repetition rate on the order of one $\mathrm{Hz}$ up to $\sim$ a kilo- $\mathrm{Hz}$ (i.e. only a small fraction of the megahertz synchrotron pulse rate). For samples with low scattering rates, multiple exposures on a single $C C D$ scan would be used in order to improve statistical accuracy. By varying the dwell time between the sample trigger and the synchrotron pulse, the sample can be probed at variable times with respect to the stimulus. The value of a $2 \mathrm{D}$ position sensitive detector with single synchrotron pulse selectivity is that it permits the measurement of a much more complete set of diffraction data; for instance, both specular and non-specular small angle scattering from thin-films and multilayers, both Bragg (specular) and diffuse scattering from bulk materials, and full diffraction patterns form liquid and amorphous materials would then be attainable. The use of an $x$-ray photocathode eliminates the long decay times of $x$-ray scintillators; however, the efficiency of photocathodes for $10 \mathrm{keV} x$-rays is only about $10 \%$. Methods for increasing hard $x$-ray efficiencies would be of significant value.
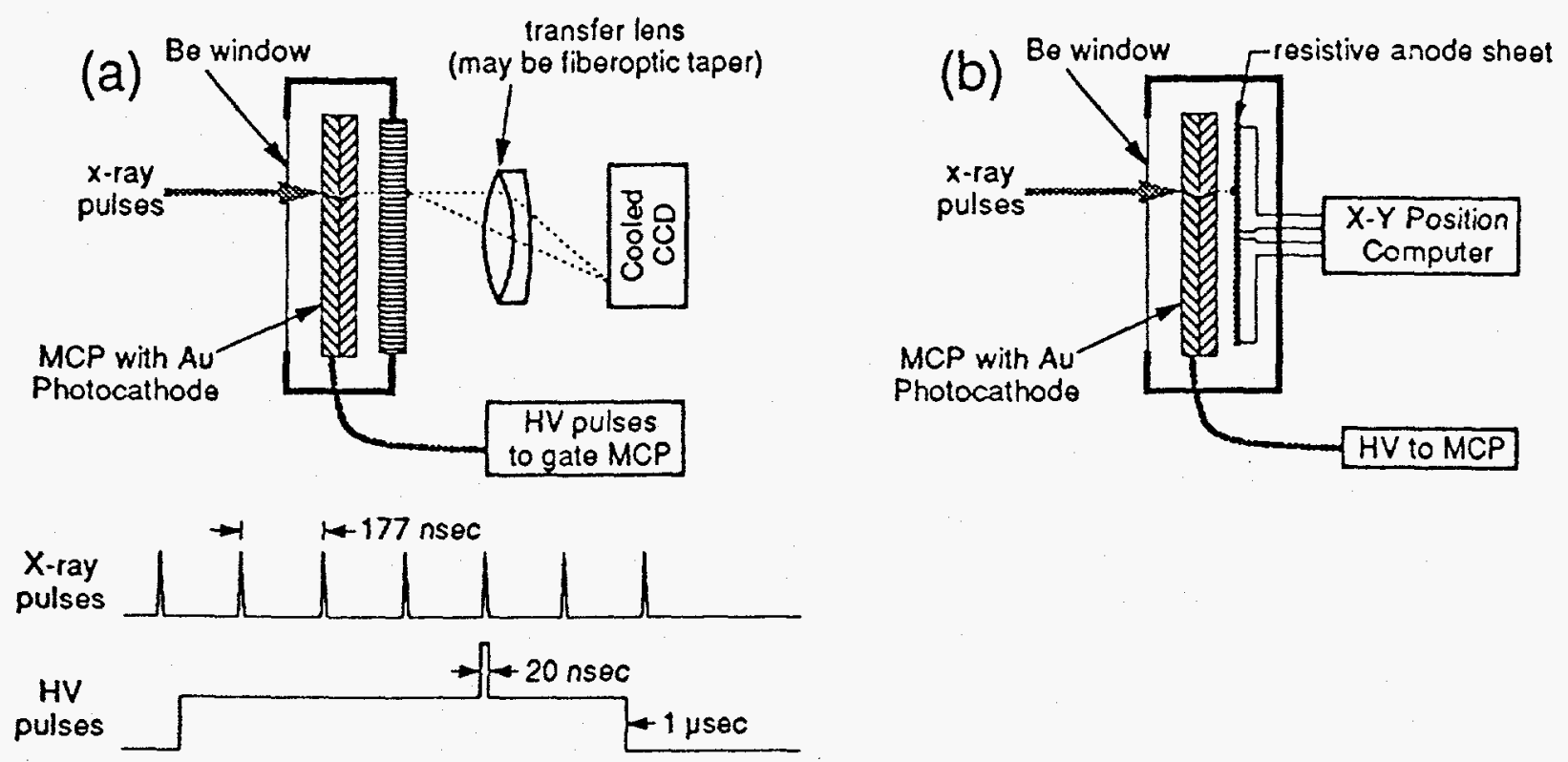

Fig. 5. (a) Pulsed microchannel plate 2D detector capable of resolving single synchrotron pulses. The scintillator output from the detector is lens or fiber-optic coupled to an integrating CCD; (b) Microchannel plate $2 \mathrm{D}$ detector recording photon arrival time and determining $x, y$ position through resistive anode.

A variation of the pulsed MCP detector in Fig. $5 \mathrm{a}$ is available ${ }^{8}$ with a resistive anode plate (Fig. $5 \mathrm{~b}$ ) rather than an optically coupled fiberoptic scintillator plate. The signals from the resistive anode plate are analyzed to determine both the position and the arrival time ${ }^{9}$ of individual photons. Although such a detector is not capable of handling simultaneous $x$-ray pulses, it does provide $2 D$ position measurements with nanosecond time resolution. The arrival time of individual photons and the low noise of such a detector make it a candidate for Mössbauer related ultra-high energy resolution $x$-ray scattering measurements that will be discussed later. 


\section{TIME RESOLYED MATERIALS SCIENCE WYYESTIGATIONS}

Time resolved structural investigations with increased resolution and higher levels of sophistication can be expected to play increasingly important roles in the development of technological materials. As materials technologies move toward the use of synthetic and nonequilibrium materials and advanced processing methods (as found in thin films, multilayers, complex composites, etc.), a higher degree of understanding and control of materials processing techniques and a knowledge of their ultimate potentials will be required. In this paper, it will not be possible to provide a comprehensive review of time-resolved materials science applications and opportunities using synchrotron $x$-ray sources. Rather, selected topics will be presented to emphasize the unique aspects and new capabilities of synchrotron sources. Examples will include investigations of transient phenomena utilizing synchrotrons and time-resolved position sensitive detectors, and they will include studies utilizing the pulsed time structure to perform ultra-high energy resolution $x$-ray scattering spectroscopy using Mössbauer resonant techniques.

\subsection{Relaxation in Strained-Layer Semiconductor Devices}

One of the ubiquitous problems associated with semiconductor devices is related to the introduction of dislocations during growth and thermal processing of multilayers. With the increased interest in Si-Ge alloys for semiconductor devices, it is becoming evident that the growth of strained layers and the introduction of dislocations is a complex process with important fundamental as well as applied questions that are unanswered. Clarke $^{5}$ et al and Lowe ${ }^{10}$ et al have used the Lemonnier 6 focusing geometry with synchrotron radiation and a virtual phase CCD operating in the streak camera mode (as depicted in Fig. 4) to investigate the thermal processing aspects of strain relaxation in multilayers. The schematic scattering pattern in Fig. 4 is illustrative of high resolution (radial) Bragg reflection profiles that Clarke, Lowe, and co-workers have made as a function of thermal heating for $\mathrm{Si}_{x} \mathrm{Ge}_{1-x}$ on $\mathrm{Si}$ and $\mathrm{In}_{x} \mathrm{Ga}_{1-x}$ As quantum well structures. 11 Through the use of this so-called streak mode, continuous angular scans of the relevant Bragg peaks for the substrate and film layers showed that strain relaxation (as observed by changes in relative Bragg peak positions) contained surprising discontinuous steps. In strained layer $\mathrm{Si}_{x} \mathrm{Ge}_{1-x}$ on $\mathrm{Si}_{1}{ }^{11}$ these discontinuities are both reversible and repeatable, leading to speculation that cooperative kinetic mechanisms rather than the kinetics of single misfit dislocations are dominant for strained layers. These effects are still under investigation.

The single line streak mode has application for time-resolved specular and non-specular small angle scattering studies and well as for grazing incidence total reflection small angle scattering measurements. While the above work was carried out in the 100 millisecond range with $20 \mu$ s row transfer times, Rodericks ${ }^{12}$ has reported advances that now allow $-5 \mu$ s row transfers $(-3 \mathrm{~ms}$ full detector readout). Therefore, this measurement scheme offers a broad range of time resolutions and provides for recording of a large number of individual rows at this rate. As discussed in the detector section above, if row transfer times of less than the single pulse spacing of the synchrotron (2.46 $\mu$ s at CHESS), the resolution of less than a nanosecond becomes possible immediately.

\subsection{In Situ Thin Film Growth Investigations}

A series of time resolved in situ thin-film growth studies were initiated by Vlieg ${ }^{13}$ et al using molecular beam epitaxy (MBE) and by Fuoss ${ }^{14}$ et al using organometallic vapor phase epitaxy (OMVPE). Rheed-like oscillations were observed in anti-Bragg truncation rod scattering as a function of surface coverage during $M B E$ growth of $G e$ on $\mathrm{Ge}(111)$ and during OMVPE deposition of $\mathrm{GaAs}$ on $\mathrm{GaAs}(100)$. The GaAs growth studies of Fuoss were carried out with $100 \mathrm{~ms}$ time resolution, with diffuse scattering howing that island spatial distributions can be determined using $\mathbf{x}$-rays, and that spatial correlations between islands are important during growth. These film growth investigations and continuing in situ growth studies of Vlieg and Fuoss and their collaborators ${ }^{13-15}$ have demonstrated that real time synchrotron $x$-ray measurements yield detailed information on kinetics and growth mechanisms. This is clearly an area with significant opportunities; pulsed film growth techniques in particular will benefit from in situ time-resolved measurements of the growth and crystallization mechanism. Figure 6 depicts a scattering configuration utilizing a gated 2D detector to carry out microsecond resolution in situ measurements during pulsed laser deposition film growth. 


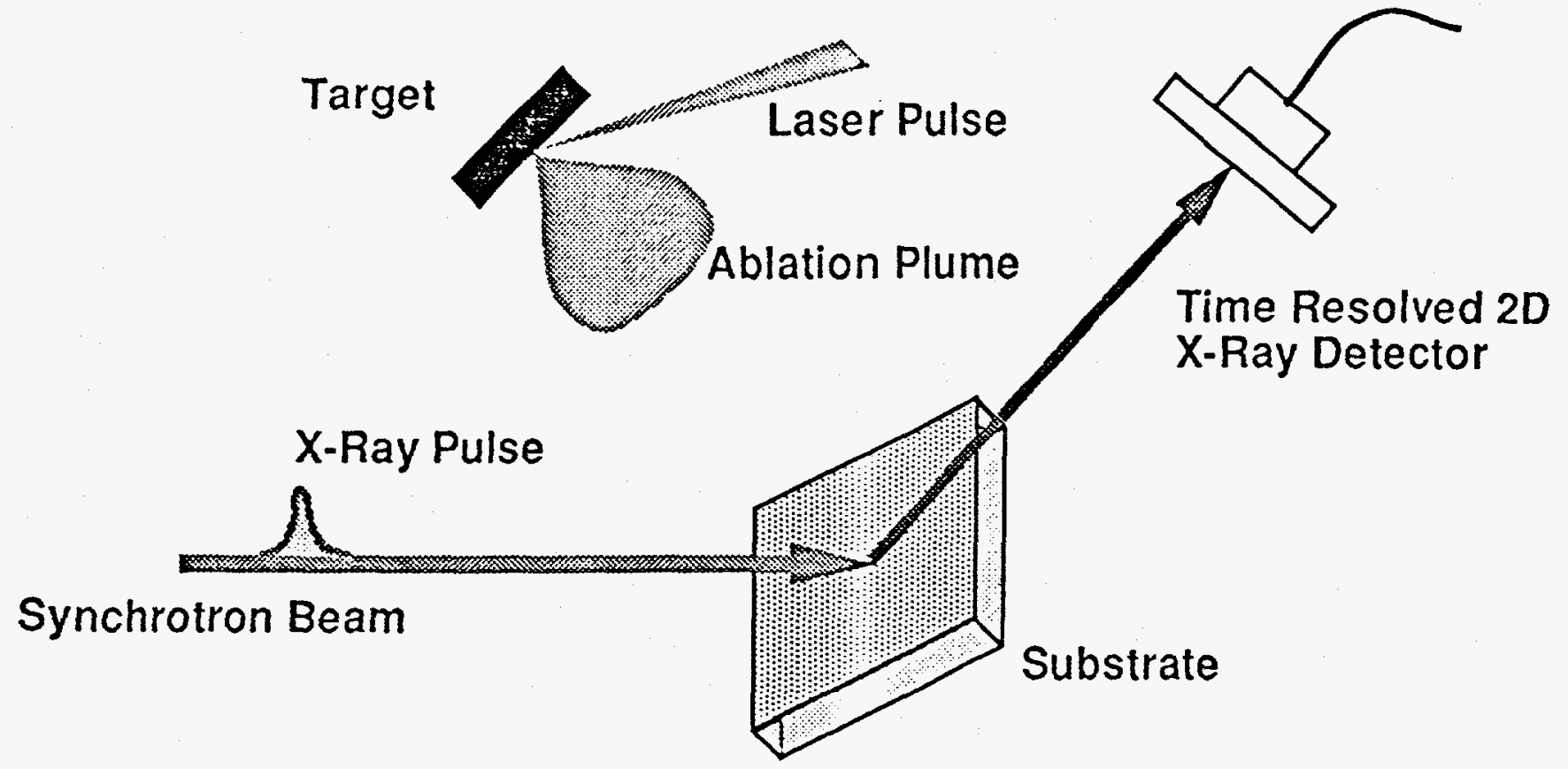

Fig. 6. Synchrotron $x$-ray scattering geometry for in situ time-resolved study of pulsed laser film growth. A nanosecond laser pulse ablates target material stoichiometrically onto a substrate, and the surface sensitive scattering is recorded by a gated 2D detector.

Twenty-five nanosecond laser pulses striking ablation targets lead to microsecond time-scale ablation plumes 16 for growth of complex high-temperature superconductor oxides as well as simple and compound semiconductors. Growth measurements from microseconds to seconds will be required to understand the entire pulsed growth process. Such measurements will be possible using the high brilliance undulator beams that will be available on the APS.

\subsection{Pulsed-Laser.Melting and Rapid Thermal Transport}

Nanosecond resolution time-resolved investigations of pulsed laser melting, regrowth, overheating, and undercooling in semiconductors have been carried out by Larson ${ }^{1,2}$ et al using a NaI detector to measure scattering from individual synchrotron $x$-ray pulses from CHESS, as shown in Fig. 7. Orientation dependent undercooling was observed during rapid $(5 \mathrm{~m} / \mathrm{s})$ regrowth of Si following pulsed laser melting. Undercooling of $11 \mathrm{~K} / \mathrm{m} / \mathrm{s}$ was found for regrowth along the $<111>$ direction, while only $5.6 \mathrm{~K} / \mathrm{m} / \mathrm{s}$ was observed for growth on $<100>$ oriented Si. An unexpected result was the absence of detectable overheating during the $10 \mathrm{~m} / \mathrm{s}$ melting process, because the principle of micro-reversibility would require a symmetry between melting and regrowth. This result suggests the possibility that the interface morphology is different for melting and regrowth.

The high brilliance of the APS and the use of pulsed 2D detectors will provide an opportunity to extend such measurements to the study of rapid thermal transport, Kapitza thermal resistance at heterointerfaces, and perhaps even structural investigations of overheated and undercooled liquid layers. Investigations of melt nucleation during picosecond laser pulses, which occurs on a time scale of tens of picoseconds in semiconductors, could be addressed using the quasi-continuous synchrotron pulse regime (Fig. 3 ) in connection with streak camera detection techniques. 


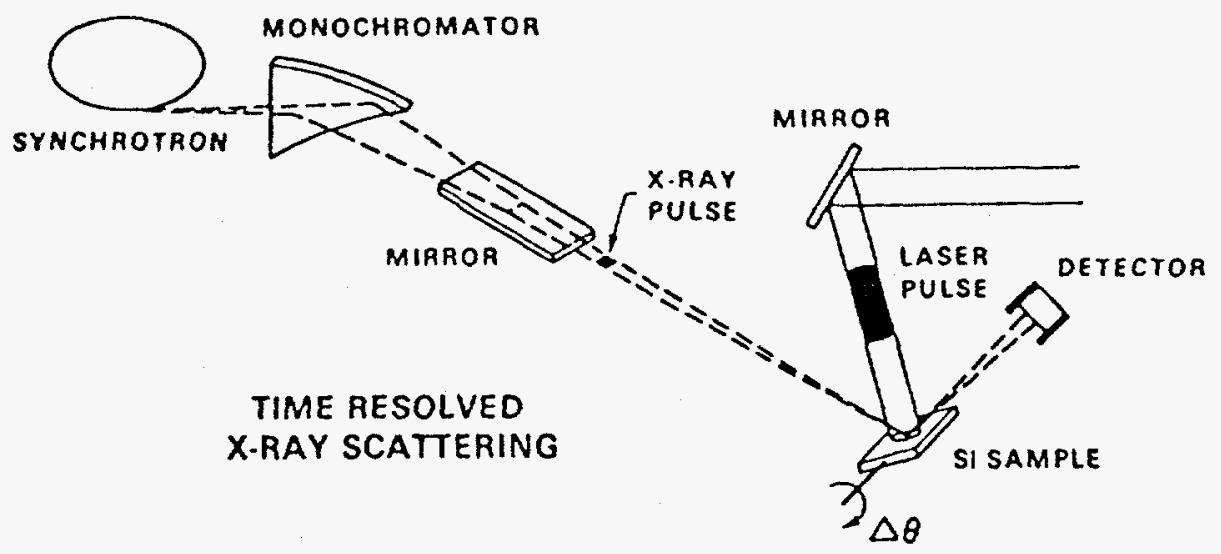

Fig. 7. Time resolved $x$-ray scattering geometry for investigation of pulsed laser melting and regrowth in which the laser pulse is synchronized with the arrival of the $x$-ray pulse and single or multiple $x$-rays scattering into the detector from a single synchrotron pulse are recorded by the pulse height in the detector.

\subsection{Time-Domain Mössbauer Resonant Spectroscopy}

The pulsed time structure of synchrotron radiation has important applications other than the investigation of transient phenomena. Figure 8a shows the delayed time-spectrum ${ }^{17}$ of Mössbauer resonant photons from a magnetic superlattice ${ }^{57} \mathrm{Fe}_{2} \mathrm{O}_{3}$ (777) reflection using the geometry in Fig. 9a. The time delay after the 150 ps synchrotron pulses separates the neV resolution resonant photons from the $\sim \mathrm{eV}$ resolution prompt electronic scattering. The physics associated with synchrotron excitation and resonant scattering is well studied, 18
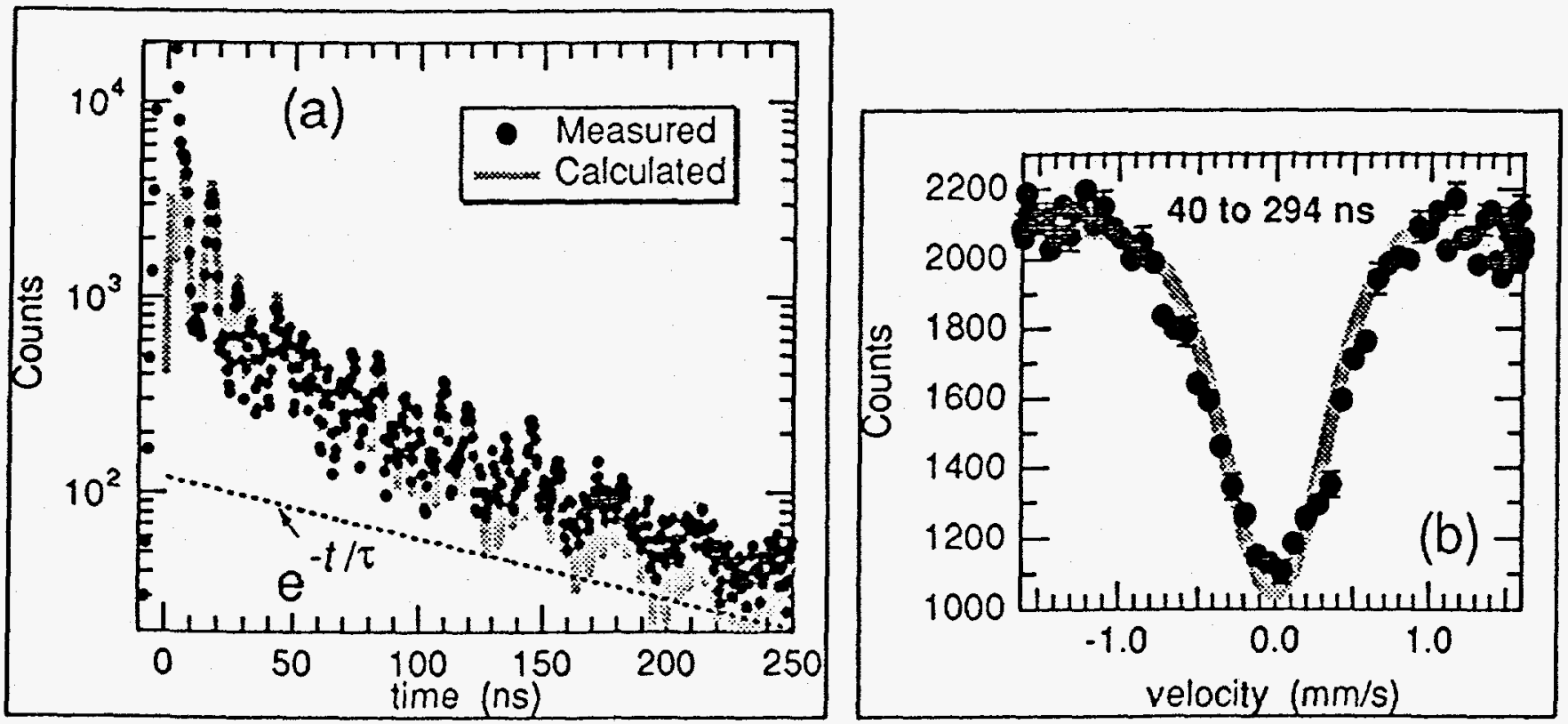

Fig. 8. (a) Delayed time-spectrum of Mössbauer resonant photons from the (777) resonant reflection of a mosaic ${ }^{57} \mathrm{Fe}_{2} \mathrm{O}_{3}$ monochromator. The time is relative to the prompt synchrotron pulse and the oscillations are a result of beating between the four hyperfine split states; (b) Doppler absorption spectrum obtained using the spectrum in (a) from 40 to $294 \mathrm{~ns}$. 
and measurements of hyperfine interaction parameters using the beat pattern, dynamical diffraction, and incoherent excitation have been performed. Figure 8 b shows a Doppler spectrum of a thick $\left(9.6 \mathrm{mg} / \mathrm{cm}^{2}\right)$ powdered ${ }^{57} \mathrm{Fe}_{2} \mathrm{O}_{3}$ absorber measured using the 40-294 $\mathrm{ns}$ interval of the resonant photon beam in Fig. 8a. Although the absorption width is relatively large $(-50 \mathrm{neV})$ because of speed up and the thick absorber, this result has polential application in neV range elastic/inelastic scattering with arc second range angular resolution. As depicted schematically in Fig. 9a, the use of a resonant monochromator and a nanosecond resolution, timeresolved 2D detector (as discussed in Fig. 5b) will provide the capability to separate elastic structural diffuse scattering from inelastic phonon scattering with high angular resolution measurements. This combination of energy and angular resolution is complementary to both neutron and conventional Mössbauer scattering spectroscopy, and is important for determining defect structures, which scalter strongly near Bragg peaks.
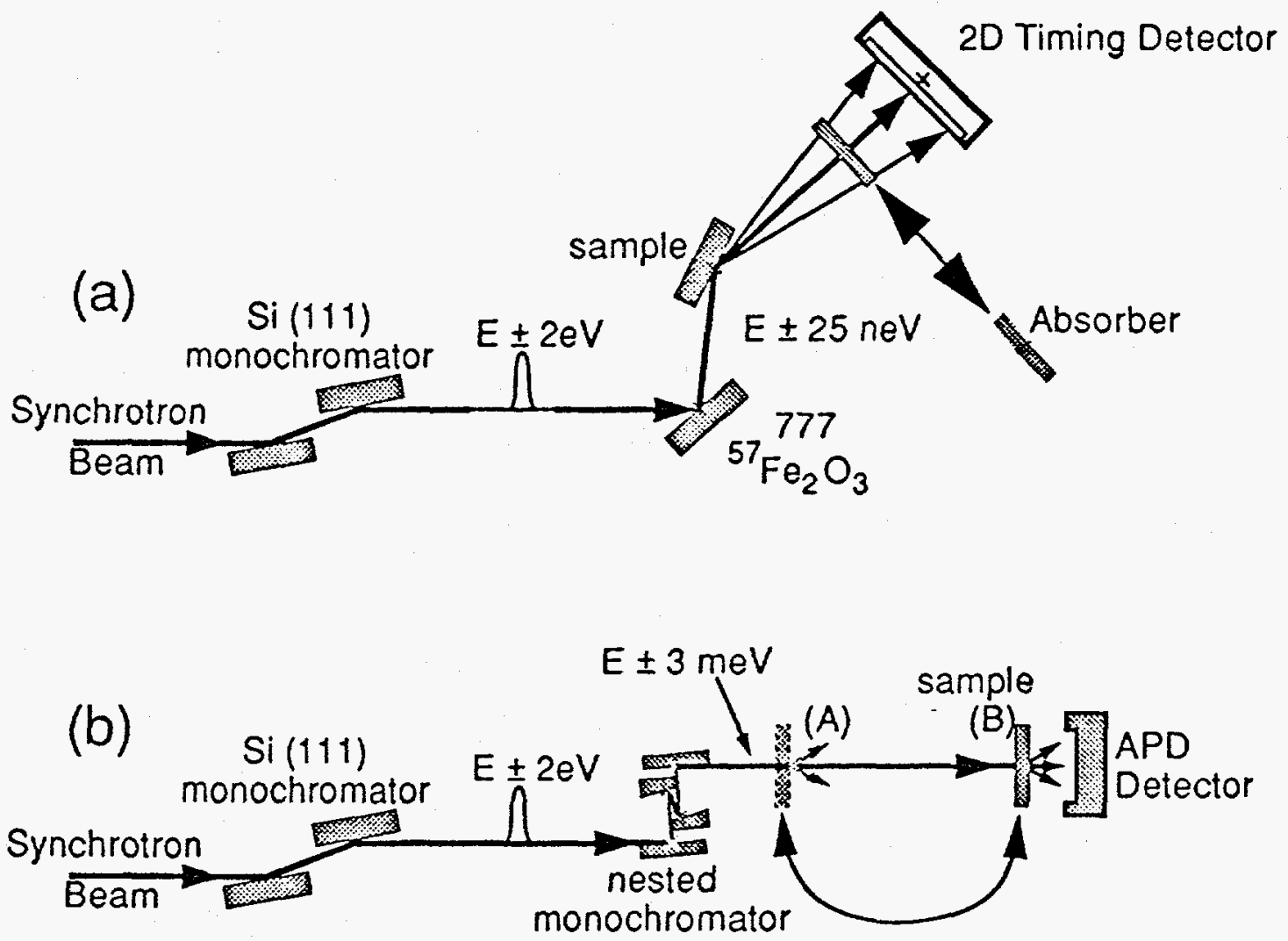

Fig. 9. (a) Geometry for Mössbauer resonant scattering spectroscopy; the scattering of resonant photons from a nonresonant sample is energy analyzed by a resonant absorber and a $2 \mathrm{D}$ timing detector. (b) Geometry for measurement of phonon density of states by scanning a 6 meV resolution x-ray beam through the Mössbauer resonant energy for ${ }^{57} \mathrm{Fe}$ enriched samples. The nested monochromator provides a scanning $6 \mathrm{meV}$ wide beam. The difference in intensity measured at sample positions $(A)$ and $(B)$ distinguishes coherent forward scattering from the incoherent scattering, which scatters into $4 \pi$ steradians.

The final application to be mentioned here is a recent development involving the pulsed time structure and Mössbauer resonance in connection with millivolt resolution monochromators to make direct measurements of the phonon density of states (DOS) in materials with resonant nuclei. Using the geometry of Fig. $9 \mathrm{~b}$, Seto ${ }^{21} \mathrm{et}^{5}$ have developed and Stuhrhahn ${ }^{22}$ et al have applied incoherent Mössbauer excitation of ${ }^{57} \mathrm{Fe}$ in $\alpha$-iron and ${ }^{57} \mathrm{Fe}$ enriched stainless steel to measure the DOS of phonons associated with Fe. Through the use of a $\sim 5 \mathrm{meV}$ resolution synchrotron $x$-ray beam varied over a range of $\pm 40 \mathrm{meV}$ around the $14.413 \mathrm{keV}{ }^{57} \mathrm{Fe}$ Mössbauer energy, these investigators were able to detect the phonon-assisted incoherent Mössbauer absorption through time-delayed monitoring of $14.4 \mathrm{keV}$ emission and delayed $\mathrm{Fe}$ fluorescence. That is, incident photons 20 millivolts away from the Mössbauer resonance will excite an ${ }^{57} \mathrm{Fe}$ nucleus only if a $20 \mathrm{meV}$ phonon is involved. Figure 10a 
depicts the (time delayed) measurement for $\alpha^{57} \mathrm{Fe}$ obtained at sample position (B) in Fig. 9b. Position (A) mensures the forward scattered coherent elastic peak (in the middle of the spectrum), so that this contribution can be rimoved from measurements at position (B) yiclding the phonon assisted spectrum as shown in Fig. 10b. This novel method is relatively fast and can be used on very small and polycrystalline samples; hence it is complementary to neutron techniques. It will also be useful for observing subtle lattice dynamics changes due to alloying, phase changes, elc. The necessity of having a Mössbauer resonant atom is somewhat restrictive, but it also provides the potential for specie specific phonon DOS in materials containing (or doped with) Mössbauer resonant nuclei.

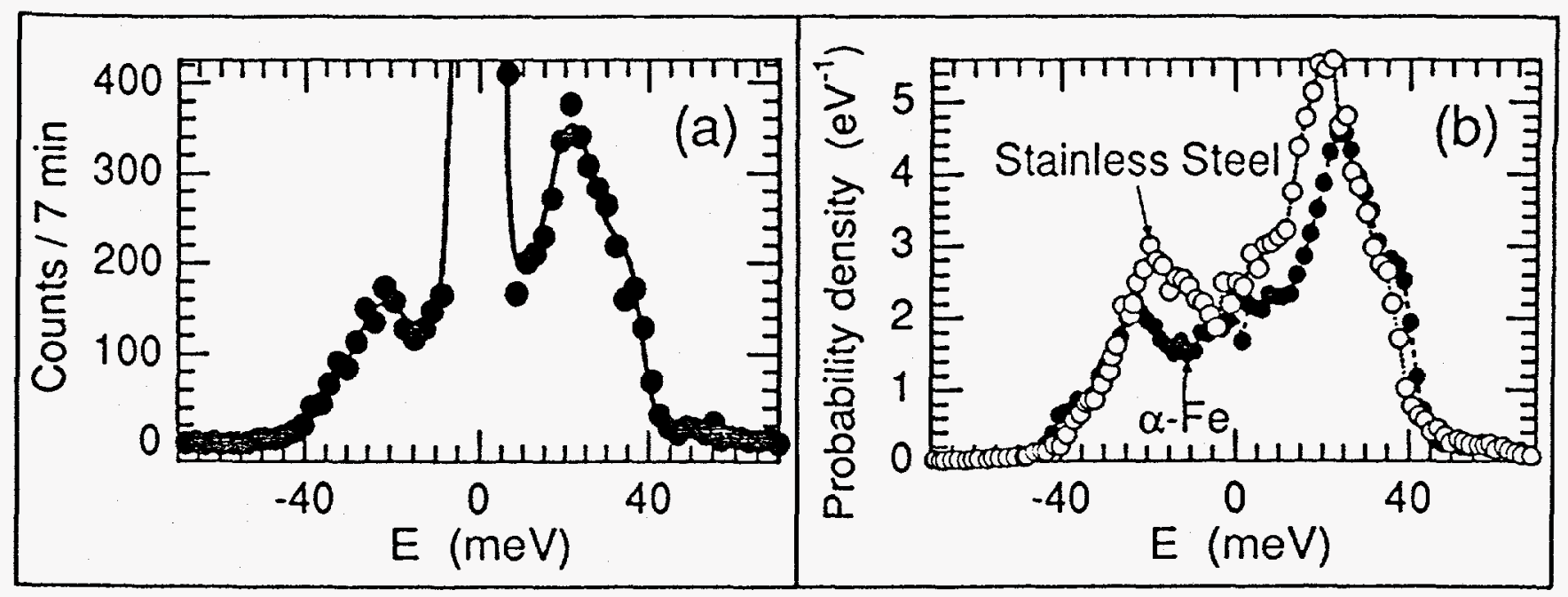

Fig. 10. Phonon assisted absorption for $\alpha-\mathrm{Fe}$, which is closely related to the phonon density of states.

\section{CONCLUSION}

Synchrotron $x$-ray sources are playing an important role in the development of comprehensive techniques for time-resolved materials science investigations. The pulsed time structure and the high intensity, in combination with the continuous energy spectrum and narrow angular collimation, provide unique opportunities for fundamental and applied investigations of the kinetics and dynamics associated with advanced materials processing techniques. The development of new time-resolved detector capabilities is having a major impact on time-resolved studies, and this trend is likely to continue. As increasing numbers of in situ real-time investigations of materials synthesis and processing are developed, the impact of synchrotron sources on advanced materials development and characterization will continue to expand.

\section{ACKNOWLEDGEMENTS}

This research was sponsored by the Division of Materials Sciences, U.S. Department of Energy, under contract No. DE-AC05-84OR21400 with Lockheed Martin Energy Systems.

\section{Z. REFERENCES}

1. B. C. Larson, J. Z. Tischler, and D. M. Mills, "Nanosecond Resolution Time-Resolved X-Ray Study of Silicon During Pulsed-Laser Irradiation," J. Mater. Res. 1 144-154 (1986), and B. C. Larson, J. Z. Tischler, and D. Mills, "Interface Temperatures and Temperature Gradients in Silicon During Pulsed-Laser Irradiation," Fundamentals of Beam-Solid Interactions and Transient Thermal Processing, M. J. Aziz, L. E. Rehn, and B. Stritzker (Editors), pp. 513-518, Materials Research Society, Pittsburgh (1988). 
2. B. C. Larson, C. W. White, T. S. Noggle, and D. M. Mills, "Synchrotron X-Ray Diffraction Study of Silicon During Pulsed-Laser Annealing," Phys. Rev. Lett. 48 337-340 (1982).

3. H. D. Bartunik, "Crystallographic Investigation of Transient States by Time-Resolved Techniques using Synchrotron Radiation," Revue Phys. Appl. 19 671-675(1984).

4. P. A. Jaanimagi, K. K. Bradley, J. Duff, G. G. Gregory, and M. C. Richardson, "Time-Resolving X-Ray Diagnostics for ICF," Rev. Sci. Instrum. 59 1854-1859 (1988).

5. R. Clarke, W. Dos Passos, W. Lowe, B. G. Rodericks, and C. Brizard, "Real-Time X-Ray Studies of Strain Kinetics in In $\mathrm{Ga}_{1-x}$ As Quantum Well Structures," Phys. Rev. Lett. $66,317-320$ (1991).

6. M. Lemonnier, R. Fourme, F. Rousseaux, and R. Kahn, Nucl. Instrum. Methods 152 , 173 (1978).

7. X-Ray and Specialty Instruments Inc., Ann Arbor, MI.

8. Quantar Technologies Incorporated, Santa Cruz, CA.

9. W. G. McMullan, S. Charbonnequ, and L. W. Thewalt, "Simultaneousl Subnanosecond Timing Information and 2D Apatial Information from Imaging Photomultiplier Tubes," Rev. Sci. Instrum. 58, 1626-1628 (1987).

10. W. Lowe, R. A. MacHarrie, J. C. Bean, L. Peticolas, R. Clark, W. Dos Passos, C. Brizzard, and B Rodricks, "RealTime X-Ray Diffraction Observation of a Pin-Slip Machanism in $\mathrm{Ge}_{x} \mathrm{Si}_{1-x}$ Strained Layers," Phys. Rev. Lett. 67 2513-2516 (1991).

11. Roy Clarke, "CCD X-Ray Detectors: Opportunities and Challenges," Nucl. Instrum. Methods A347, 529-533 (1994).

12. Brian Rodericks, "Time-Resolved X-Ray Xcattering Program at the Advanced Photon Source," Rev. Sci. Instrum. $\underline{66}, 1456-1458$ (1995).

13. E. Vlieg, A. W. Denier van der Gon, J. F. van der Veen, J. E. Macdonald, and C. Norris, "Surface X-Ray Scattering During Crystal Growth: Ge on Ge(111)," Phys. Rev. Lett. 61, 2241-2244 (1988).

14. P. H. Fuoss, D. W. Kisker, F. J. Lamelas, G. B. Stephenson, P. Imperatori, and S. Brennan, "Time-Resolved $X$-Ray Scattering Studies of Layer-by-Layer Epitaxial Growth," Phys. Rev. Lett. 69 2791-2794 (1992).

15. F. J. Lamelas, P. H. Fuoss, D. W. Kisker, G. B. Stephenson, P. Imperatori, and S. Brennan, "X-Ray Scaltering Analysis of Surface Structures Produced by Vapor-Phase Epitaxy of GaAs," Phys. Rev. B49, 1957-1965 (1994).

16. D. B. Geohegan, Appl. Phys. Lett. 602732 (1992).

17. J. Z. Tischler, B. C. Larson, G. E. Ice, and P. Zschack, "Time Structure and Mössbauer Filtering of Nuclear Bragg Scatteing from Amosaic ${ }^{57} \mathrm{Fe}_{2} \mathrm{O}_{3}$ Crystal," Phys. Rev. B47 552 (1993).

18. G. Faigel, D. P. Siddons, J. B. Hastings, P. E. Haustein, and J. R. Grover, "New Approach to the Study of Nuclear Bragg Scattering of Synchrotron Radiation," Phys. Rev. Lett. 58, 2699-2701 (1987), and G. Faigel, D. P. Siddons, J. B. Hastings, P. E. Haustein, J. R. Grover, and L. E. Berman, "Observation of th Full Time Evolution

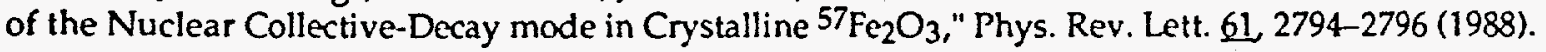


19. E. Gerdau, R. Rüffer, R. Hollatz, and J. P. Hannon, "Quantum Beats from Nuclei Excited by Synchrotron Radiation," Phys. Rev. Lett. 57 1141-1144 (1986), and U. Van Burck, R. L. Mössbauer, E. Gerdau, R. Rüffer, R. Hollatz, G. V. Smimov, and J. P. Hannon, "Nuclear Bragg Scattering of Synchrotron Radiation with Strong Speed up of Coherent Decay, Measured on Anti-ferromagnetic 57 FeBO3," Phys. Rev. Lett. 59 335-338 (1987).

20. E. E. Alp, T. M. Mooney, T. Toellner, W. Sturhahn, E. Witthoff, R. Röhlsberger, E. Gerdau, H. Homma, and M. Kentjana, "Time Resolved NuclearResonant Scattering from ${ }^{119}$ ISn Nuclei using Synchrotron Radiation," Phys. Rev. Lett. 70, 3351-3354, (1993).

21. M. Seta, Y. Yoda, S. Kikuta, X. W. Zhang, and M. Ando, "Observation of Nuclear Resonant Scattering Accompanied by Phonon Excitation Using Synchrotron Radiation," Phys. Rev. Lett. 74 3828-3831 (1995).

22. W. Stuhrhahn, T. S. Toellner, E. E. Alp, X. Zhang, M. Ando, Y. Yoda, S. Kikuta, M. Seto, C. W. Kimball, and B. Dabrowski, "Thonon Density of States Measured by Inelastic Nuclear Resonant Scattering," Phys. Rev. Lett. 74, 3832-3835 (1995). 